\title{
Transmuted Weibull distribution and its applications
}

\author{
Ivana Pobočíková ${ }^{1}$, Zuzana Sedliačková ${ }^{1}$, Mária Michalková ${ }^{1, *}$ \\ ${ }^{1}$ Department of Applied Mathematics, Faculty of Mechanical Engineering, University of Žilina, \\ Univerzitná 1, 01026 Žilina, Slovakia
}

\begin{abstract}
In this paper we study new distribution called transmuted Weibull distribution. Some properties of this distribution are described. The usefulness of the distribution for modelling data is illustrated using real data set.
\end{abstract}

Keywords: transmuted Weibull distribution, properties, maximum likelihood estimation, application; Akaike's information criterion, Bayesian information criterion, coefficient of determination, root mean square error

\section{Introduction}

The Weibull distribution was originally introduced by Swedish physicist Waloddi Weibull [1]. He applied it on modelling the distribution of the yield strength of materials. The Weibull distribution is popular and widely used, with many applications in areas such as engineering (reliability, failure analysis, lifetime analysis ...), material science, quality control, physics, medicine, meteorology, hydrology and others [2].

However, there are cases when standard Weibull distribution fails to model data suitably enough. This is where it is necessary to apply generalized distribution because of its flexibility and better fit of the data than standard Weibull distribution. The importance of such generalization has been proved in recent years on various problems and many standard distributions have been generalized. Some recent generalizations which are based on the Weibull distribution, are discussed in [3].

An interesting idea of generalization where the distribution is derived using the quadratic rank transmutation map, was studied first by Shaw and Buckley in [4]. Recently, many transmuted distributions have been proposed in literature. See, for example, transmuted Gumbel distribution [5], transmuted Rayleigh distribution [6], transmuted modified Weibull distribution [7], transmuted inverse Weibull distribution [8], transmuted Pareto distribution [9] and others.

Aryal and Tsokos [10] used the quadratic rank transmutation map to develop new generalization of the Weibull distribution, called the transmuted Weibull distribution. They studied mathematical properties of this distribution and the maximum likelihood estimates of the parameters.

\footnotetext{
*Corresponding author: maria.michalkova@fstroj.uniza.sk

Reviewers: Vladimír Dekýš, Urszula Siedlecka
} 
In this paper, we focus on demonstrating the usefulness of the transmuted Weibull distribution for modelling lifetime, illustrated on three real data sets. All three data sets are modelled with the transmuted Weibull distribution, the 2-parameter and 3-parameter Weibull distribution. For comparison of the distributions the following criteria are used: the Akaike's information criterion, the corrected Akaike's information criterion, the Bayesian information criterion, the coefficient of determination and the root mean square error.

The parameters of the probability distributions are estimated using the maximum likelihood method. The calculations are performed using statistical software STATISTICA and software MATLAB.

The rest of the paper is organized as follows: In Section 2 we introduce the transmuted Weibull distribution. In Section 3 we define basic statistical properties including moments, quantiles, reliability and hazard rate functions. The maximum likelihood estimates of the transmuted Weibull parameters are presented in Section 4. Finally, in Section 5 we provide the real data applications of the transmuted Weibull distribution.

\section{Transmuted Weibull distribution}

A random variable $X$ is said to have the 3-parameter Weibull distribution (WD) $W(a, b, c)$ with parameters $a>0, b>0, c \geq 0$ if its cumulative distribution function (CDF) for $x \geq c$ is given by

$$
G(x)=1-\exp \left(-\left(\frac{x-c}{b}\right)^{a}\right)
$$

and probability density function (PDF) is given by

$$
g(x)=\frac{a}{b^{a}}(x-c)^{a-1} \exp \left(-\left(\frac{x-c}{b}\right)^{a}\right)
$$

where $a$ is the dimensionless shape parameter, $b$ is the scale parameter and $c$ is the location parameter.

Setting $c=0$, (1) and (2) transform into CDF and PDF, respectively, of the 2-parameter Weibull distribution $W(a, b)$.

The transmuted distribution can be obtained by adding real number $\lambda,|\lambda| \leq 1$, into CDF that provides more flexibility in the form of new distribution [7]. $\lambda$ denotes the transmuting parameter.

A random variable $X$ is said to have the transmuted distribution if its $\mathrm{CDF} F(x)$ and PDF $f(x)$ are given by

$$
\begin{gathered}
F(x)=(1+\lambda) G(x)-\lambda G(x)^{2} \\
f(x)=g(x)[(1+\lambda)-2 \lambda G(x)]
\end{gathered}
$$

where $|\lambda| \leq 1, G(x)$ and $g(x)$ are CDF and PDF of the base distribution, respectively. Observe that for $\lambda=0$ we have the base distribution of the random variable $X$.

Let us consider the 2-parameter Weibull distribution. Adding (1) into (3) we have CDF of the transmuted Weibull distribution (TWD) $W(a, b, \lambda)$ with parameters $a>0, b>0$ and $|\lambda| \leq 1$ 


$$
F(x)=\left[1-\exp \left(-\left(\frac{x}{b}\right)^{a}\right)\right]\left[1+\lambda \exp \left(-\left(\frac{x}{b}\right)^{a}\right)\right]
$$

Similarly, combining (2), (3) and (4) we have PDF of the TWD

$$
f(x)=\frac{a}{b^{a}} x^{a-1} \exp \left(-\left(\frac{x}{b}\right)^{a}\right)\left[1-\lambda+2 \lambda \exp \left(-\left(\frac{x}{b}\right)^{a}\right)\right]
$$

\section{Statistical properties}

In this section we define statistical properties including moments, quantiles, reliability and hazard rate functions. The origin and other aspects of this distribution can be found in [10].

\subsection{Moments and quantiles}

The $r$-th moment, $r=1,2, \ldots$, of the TWD is defined as

$$
E\left(X^{r}\right)=b^{r} \Gamma\left(\frac{r}{a}+1\right)\left[1-\lambda+\lambda 2^{-r / a}\right]
$$

Consequently, the mean $E(X)$ and the variance $\operatorname{Var}(X)$ are given by

$$
\begin{gathered}
E(X)=b \Gamma\left(\frac{1}{a}+1\right)\left[1-\lambda+\lambda 2^{-1 / a}\right] \\
\operatorname{Var}(X)=b^{2}\left\{\Gamma\left(\frac{2}{a}+1\right)\left[1-\lambda+\lambda 2^{-2 / a}\right]-\Gamma^{2}\left(\frac{1}{a}+1\right)\left[1-\lambda+\lambda 2^{-1 / a}\right]^{2}\right\}
\end{gathered}
$$

respectively, where $\Gamma(p)$ is gamma function defined by $\Gamma(p)=\int_{0}^{\infty} x^{p-1} e^{-x} d x, p>0$. The $\alpha$-th quantile, $\alpha \in(0,1)$, of the TWD is given by

$$
x_{\alpha}=b\left[-\ln \left(1-\left(\frac{1+\lambda-\sqrt{(1+\lambda)^{2}-4 \lambda \alpha}}{2 \lambda}\right)\right)\right]^{1 / a}
$$

\subsection{Reliability analysis}

The TWD can be useful in characterization of the lifetime data analysis. The reliability function if the TWD is given by

$$
R(x)=\exp \left(-\left(\frac{x}{b}\right)^{a}\right)\left[1-\lambda+\lambda \exp \left(-\left(\frac{x}{b}\right)^{a}\right)\right]
$$

This function can be interpreted as probability that an item does not fail prior to some time $x$.

Another important characteristics is the hazard rate function that can be interpreted as conditional probability of failure when the item has already survived to time $x$. 
The hazard rate function of the TWD is given by

$$
H(x)=\frac{a}{b^{a}} x^{a-1}\left[\frac{1-\lambda+2 \lambda \exp \left(-\left(\frac{x}{b}\right)^{a}\right)}{1-\lambda+\lambda \exp \left(-\left(\frac{x}{b}\right)^{a}\right)}\right]
$$

\subsection{Random number generation}

The random numbers from the TWD can be generated using the inverse function of CDF (5)

$$
X=b\left[-\ln \left(1-\left(\frac{1+\lambda-\sqrt{(1+\lambda)^{2}-4 \lambda U}}{2 \lambda}\right)\right)\right]^{1 / a}
$$

where $U$ has uniform distribution over the interval $(0,1)$.

\section{Maximum likelihood estimates}

As a method for estimation of parameters of the TWD we choose the maximum likelihood method (MLM).

Let $X_{1}, X_{2}, \ldots, X_{n}$ be a random sample of size $n$ from the TWD with PDF (6) and let $x_{1}, x_{2}, \ldots, x_{n}$ be a realization of the random sample. The likelihood function $L=L\left(x_{1}, x_{2}, \ldots, x_{n} ; a, b, \lambda\right)$ of this random sample is given by

$$
L=\left(\frac{a}{b^{a}}\right)^{n} \exp \left(-\frac{1}{b^{a}} \sum_{i=1}^{n} x_{i}^{a}\right) \prod_{i=1}^{n} x_{i}^{a-1}\left[1-\lambda+2 \lambda \exp \left(-\left(\frac{x_{i}}{b}\right)^{a}\right)\right]
$$

and the $\log$-likelihood function $\ln L=\ln L\left(x_{1}, x_{2}, \ldots, x_{n} ; a, b, \lambda\right)$ of this random sample is given by

$$
\ln L=n \ln \frac{a}{b^{a}}-\frac{1}{b^{a}} \sum_{i=1}^{n} x_{i}^{a}+(a-1) \sum_{i=1}^{n} \ln x_{i}+\sum_{i=1}^{n} \ln \left[1-\lambda+2 \lambda \exp \left(-\left(\frac{x_{i}}{b}\right)^{a}\right)\right]
$$

Differentiating (15) with respect to $a, b$ and $\lambda$, respectively, and equating each derivative to zero we obtain the equations

$$
\begin{gathered}
\frac{n}{a}+\sum_{i=1}^{n} \ln \left(\frac{x_{i}}{b}\right)\left[1-\left(\frac{x_{i}}{b}\right)^{a}\right]-2 \lambda \sum_{i=1}^{n} \frac{\left(\frac{x_{i}}{b}\right)^{a} \ln \left(\frac{x_{i}}{b}\right) \exp \left(-\left(\frac{x_{i}}{b}\right)^{a}\right)}{1-\lambda+2 \lambda \exp \left(-\left(\frac{x_{i}}{b}\right)^{a}\right)}=0 \\
\frac{a}{b} \sum_{i=1}^{n}\left[\left(\frac{x_{i}}{b}\right)^{a}-1\right]-2 \lambda \frac{a}{b} \sum_{i=1}^{n} \frac{\left(\frac{x_{i}}{b}\right)^{a} \exp \left(-\left(\frac{x_{i}}{b}\right)^{a}\right)}{1-\lambda+2 \lambda \exp \left(-\left(\frac{x_{i}}{b}\right)^{a}\right)}=0
\end{gathered}
$$




$$
\sum_{i=1}^{n} \frac{2 \exp \left(-\left(\frac{x_{i}}{b}\right)^{a}\right)-1}{1-\lambda+2 \lambda \exp \left(-\left(\frac{x_{i}}{b}\right)^{a}\right)}=0
$$

The MLM estimates $\hat{a}, \hat{b}, \hat{\lambda}$ of the parameters $a, b, \lambda$ are obtained iteratively from the nonlinear system of equations (16) - (18).

\section{Application}

In this section we present the analysis of the real data sets using the TWD and compare it with the WD. In order to compare the distributions we consider the criteria:

- the Akaike's information criterion $(A I C)$ - calculated using [11]

$$
A I C=-2 \ln L(\hat{\theta})+2 m
$$

where $L(\hat{\theta})=L\left(x_{1}, x_{2}, \ldots, x_{n} ; \hat{\theta}\right)$ is the maximized value of the likelihood function for the estimated model, $\hat{\theta}$ is the MLM estimate of the parameter $\theta, m$ is number of parameters to be estimated, $n$ is number of observed data;

- the corrected Akaike's information criterion (AICC) - calculated using [12]

$$
A I C C=A I C+\frac{2 m(m+1)}{n-m+1}
$$

- the Bayesian information criterion $(B I C)$ - calculated using [13]

$$
B I C=-2 \ln L(\hat{\theta})+m \ln (n) ;
$$

- the coefficient of determination $\left(R^{2}\right)$ - calculated using

$$
R^{2}=\frac{\sum_{i=1}^{n}\left(\hat{F}\left(x_{i}\right)-\bar{F}\right)^{2}}{\sum_{i=1}^{n}\left(\hat{F}\left(x_{i}\right)-\bar{F}\right)^{2}+\sum_{i=1}^{n}\left(F_{n}\left(x_{i}\right)-\hat{F}\left(x_{i}\right)\right)^{2}}
$$

where $\widehat{F}(x)$ is estimated cumulative distribution function and

$$
\bar{F}=\frac{1}{n} \sum_{i=1}^{n} \hat{F}\left(x_{i}\right)
$$

Function $F_{n}(x)$ is the empirical distribution function, defined as follows

$$
F_{n}(x)=\frac{1}{n} \sum_{i=1}^{n} I\left(x_{(i)} \leq x\right)
$$

where $I\left(x_{(i)} \leq x\right)=1$ if $x_{(i)} \leq x$ and 0 otherwise, $x_{(1)}, x_{(2)}, \ldots, x_{(n)}$ are observations in ascending order, i.e. $x_{(1)} \leq x_{(2)} \leq \cdots \leq x_{(n)}$; 
- the root mean square error (RMSE) - calculated using

$$
R M S E=\left[\frac{1}{n} \sum_{i=1}^{n}\left(F_{n}\left(x_{i}\right)-\hat{F}\left(x_{i}\right)\right)^{2}\right]^{1 / 2}
$$

The distribution better fitting the data corresponds to smaller values of $A I C, B I C, A I C C$ and $R M S E$ and larger value of $R^{2}$.

\section{Data set 1}

The first data set represents lifetimes of Kevlar 49/epoxy strands subjected to constant sustained pressure at $90 \%$ stress level until the strand failure. For previous studies with the data see $[14-17]$. The data are as follows:

$0.0251,0.0886,0.0891,0.2501,0.3113,0.3451,0.4763,0.5650,0.5671,0.6566$, $0.6748,0.6751,0.6753,0.7696,0.8375,0.8391,0.8425,0.8645,0.8851,0.9113$, $0.9120,0.9836,1.0483,1.0596,1.0773,1.1733,1.2570,1.2766,1.2985,1.3211$, $1.3503,1.3551,1.4595,1.4880,1.5728,1.5733,1.7083,1.7263,1.7460,1.7630$, $1.7746,1.8275,1.8375,1.8503,1.8808,1.8878,1.8881,1.9316,1.9558,2.0048$, $2.0408,2.0903,2.1093,2.1330,2.2100,2.2460,2.2878,2.3203,2.3470,2.3513$, $2.4951,2.5260,2.9911,3.0256,3.2678,3.4045,3.4846,3.7433,3.7455,3.9143$, $4.8073,5.4005,5.4435,5.5295,6.5541,9.0960$.

Table 1 gives the descriptive statistics for the data set. We can see that the empirical distribution is skewed to the right. Table 2 presents the MLM estimates of the parameters together with the log-likelihood $\ln L, A I C, A I C C$ and $B I C$ values. Results are rounded to four decimal places.

Table 1. Descriptive statistics for data set 1

\begin{tabular}{|c|c|c|c|c|c|c|c|c|}
\hline Min & Max & Mean & Variance & $\begin{array}{c}\text { Lower } \\
\text { Quantile }\end{array}$ & Median & $\begin{array}{c}\text { Upper } \\
\text { Quantile }\end{array}$ & Skewness & Kurtosis \\
\hline 0.0251 & 9.0960 & 1.9592 & 2.4774 & 0.8982 & 1.7362 & 2.3041 & 2.0196 & 5.6004 \\
\hline
\end{tabular}

Table 2. MLM estimates of the parameters and model selection criteria for data set 1

\begin{tabular}{|c|c|c|c|c|c|c|c|}
\hline \multirow{2}{*}{\begin{tabular}{|c|} 
Distributio \\
$n$ \\
\end{tabular}} & \multirow{2}{*}{$\begin{array}{c}\text { Parameter } \\
\text { estimates }\end{array}$} & \multicolumn{6}{|c|}{ Statistical test } \\
\hline & & $\ln L$ & $A I C$ & $A I C C$ & $B I C$ & $R^{2}$ & RMSE \\
\hline$W(a, b)$ & $\begin{array}{l}\hat{a}=1.3256 \\
\hat{b}=2.1328\end{array}$ & -122.5247 & 249.0494 & 249.2094 & 253.7108 & 0.9753 & 0.0423 \\
\hline$W(a, b, c)$ & $\begin{array}{l}\hat{a}=1.3169 \\
\hat{b}=2.1228 \\
\hat{c}=0.0058\end{array}$ & -122.5141 & 251.0282 & 251.3525 & 258.0204 & 0.9750 & 0.0425 \\
\hline$W(a, b, \lambda)$ & $\begin{array}{l}\hat{a}=1.0509 \\
\hat{b}=1.4419 \\
\hat{\lambda}=-0.7955\end{array}$ & -121.4300 & 248.8600 & 249.1843 & 255.8522 & 0.9838 & 0.0351 \\
\hline
\end{tabular}

In Table 2 we see that values of $A I C, A I C C$ and $R M S E$ are smallest ones and value of $\ln L$ and $R^{2}$ are highest ones for TWD, compared to values for 2-parameter and 3parameter WDs. Hence, we can conclude that the TWD provides better fit to the data than the other two distributions. 


\section{Data set 2}

The second data set represents survival times (in days) of 72 guinea pigs infected with virulent tubercle bacilli. The data were observed and reported in [18]. For previous studies with the data see [19]. The data are as follows:

$10,33,44,56,59,72,74,77,92,93,96,100,100,102,105,107,107$, $108,108,108,109,112,113,115,116,120,121,122,122,124,130,134,136,139$, $144,146,153,159,160,163,163,168,171,172,176,183,195,196,197,202,213$, $215,216,222,230,231,240,245,251,253,254,254,278,293,327,342,347,361$, 402, 432, 458, 555 .

Table 3 gives the descriptive statistics for data set 2 . The value of skewness indicates that the empirical distribution is skewed to the right. Table 4 presents the MLM estimates of the parameters and criteria for comparison of TWD with 2-parameter and 3-parameter WDs.

Table 3. Descriptive statistics for data set 2

\begin{tabular}{|c|c|c|c|c|c|c|c|c|}
\hline Min & Max & Mean & Variance & $\begin{array}{c}\text { Lower } \\
\text { Quantile }\end{array}$ & Median & $\begin{array}{c}\text { Upper } \\
\text { Quantile }\end{array}$ & Skewness & Kurtosis \\
\hline 10 & 555 & 176.8333 & 10705.0986 & 108 & 149.5 & 226 & 1.3700 & 2.2221 \\
\hline
\end{tabular}

Table 4. MLM estimates for the parameters and model selection criteria for data set 2

\begin{tabular}{|c|c|c|c|c|c|c|c|}
\hline \multirow{2}{*}{ Distribution } & \multirow{2}{*}{$\begin{array}{c}\text { Parameter } \\
\text { estimates }\end{array}$} & \multicolumn{6}{|c|}{ Statistical test } \\
\hline & & $\ln L$ & AIC & $A I C C$ & $B I C$ & $R^{2}$ & RMSE \\
\hline$W(a, b)$ & $\begin{array}{l}\hat{a}=1.8253 \\
\hat{b}=199.6177\end{array}$ & -427.3700 & 858.7400 & 858.9090 & 863.2933 & 0.9694 & 0.0477 \\
\hline$W(a, b, c)$ & $\begin{array}{l}\hat{a}=1.7610 \\
\hat{b}=193.3730 \\
\hat{c}=5.0001\end{array}$ & -427.1846 & 860.3692 & 860.7121 & 867.1992 & 0.9711 & 0.0463 \\
\hline$W(a, b, \lambda)$ & $\begin{array}{l}\hat{a}=1.3534 \\
\hat{b}=139.6319 \\
\hat{\lambda}=-0.9506\end{array}$ & -425.6935 & 857.3870 & 857.7299 & 864.2170 & 0.9814 & 0.0382 \\
\hline
\end{tabular}

Values in Table 4 indicate that the TWD provides better fit to the data than the 2 parameter and 3-parameter WDs.

\section{Data set 3}

The third data set represents age of 155 patients suffering from the breast cancer, recorded from June to November 2014, who entered the Breast Tumours Detection Unit, Benha Hospital University, Egypt. The data were observed and reported in [20]. The data are as follows:

$12,17,18,18,20,24,24,25,26,28,28,28,29,30,30,30,30,31,31,31,32,32,32,32$, $33,34,34,35,35,35,35,35,35,35,36,36,36,36,36,36,38,38,38,38,38,38,38,38$, $38,38,38,38,38,39,39,40,40,40,40,40,40,40,40,40,40,40,40,40,40,40,40,40$, $41,42,42,42,42,42,42,42,42,43,43,44,44,45,45,45,45,45,45,45,46,46,47,48$, $48,48,48,48,48,48,49,49,50,50,50,50,50,50,50,50,50,50,50,50,50,50,50,50$, $50,50,50,50,50,50,50,52,52,52,53,54,54,55,56,56,58,58,58,59,60,60,60,60$, $60,60,60,63,65,65,66,69,80,90,90$. 
Table 5 gives the descriptive statistics for data set 3. Table 6 presents the MLM estimates of the parameters and the comparison of TWD with the 2-parameter and 3parameter WDs.

Table 5. Descriptive statistics for data set 3

\begin{tabular}{|c|c|c|c|c|c|c|c|c|}
\hline Min & Max & Mean & Variance & $\begin{array}{c}\text { Lower } \\
\text { Quantile }\end{array}$ & Median & $\begin{array}{c}\text { Upper } \\
\text { Quantile }\end{array}$ & Skewness & Kurtosis \\
\hline 12 & 90 & 43.6516 & 146.0597 & 36 & 42 & 50 & 0.6915 & 2.2738 \\
\hline
\end{tabular}

Table 6. MLM estimates and model selection criteria for data set 3

\begin{tabular}{|c|c|c|c|c|c|c|c|}
\hline \multirow{2}{*}{ Distribution } & \multirow{2}{*}{$\begin{array}{c}\text { Parameter } \\
\text { estimates }\end{array}$} & \multicolumn{6}{|c|}{ Statistical test } \\
\hline & & \begin{tabular}{|l|}
$\ln L$ \\
\end{tabular} & $A I C$ & $A I C C$ & $B I C$ & $R^{2}$ & RMSE \\
\hline$W(a, b)$ & $\begin{array}{l}\hat{a}=3.6871 \\
\hat{b}=48.1100\end{array}$ & -610.2967 & 1224.5934 & 1224.6713 & 1230.6802 & 0.9640 & 0.0491 \\
\hline$W(a, b, c)$ & $\begin{array}{l}\hat{a}=2.9515 \\
\hat{b}=38.3366 \\
\hat{c}=9.2768\end{array}$ & -607.4815 & 1220.9629 & 1221.1189 & 1230.0932 & 0.9656 & 0.0489 \\
\hline$W(a, b, \lambda)$ & $\begin{array}{l}\hat{a}=4.0312 \\
\hat{b}=55.0570 \\
\hat{\lambda}=0.8380\end{array}$ & -605.5600 & 1217.1200 & 1217.2768 & 1226.2502 & 0.9733 & 0.0438 \\
\hline
\end{tabular}

Values in Table 6 indicate that the TWD provides better fit to data than the 2-parameter and 3-parameter WDs.

Figures 1, 2 and 3 illustrate the histograms and the fitted PDFs of the TWD, 2parameter WD and 3-parameter WD for data set 1, 2 and 3, respectively.

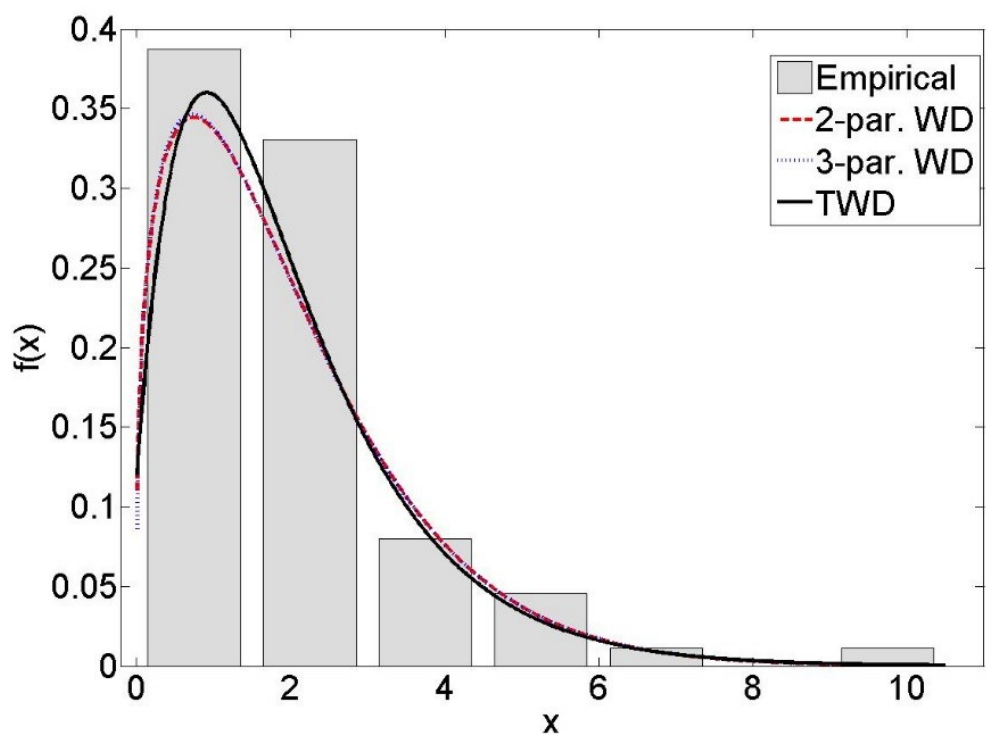

Fig. 1. Histogram of data set 1 and the fitted PDFs 


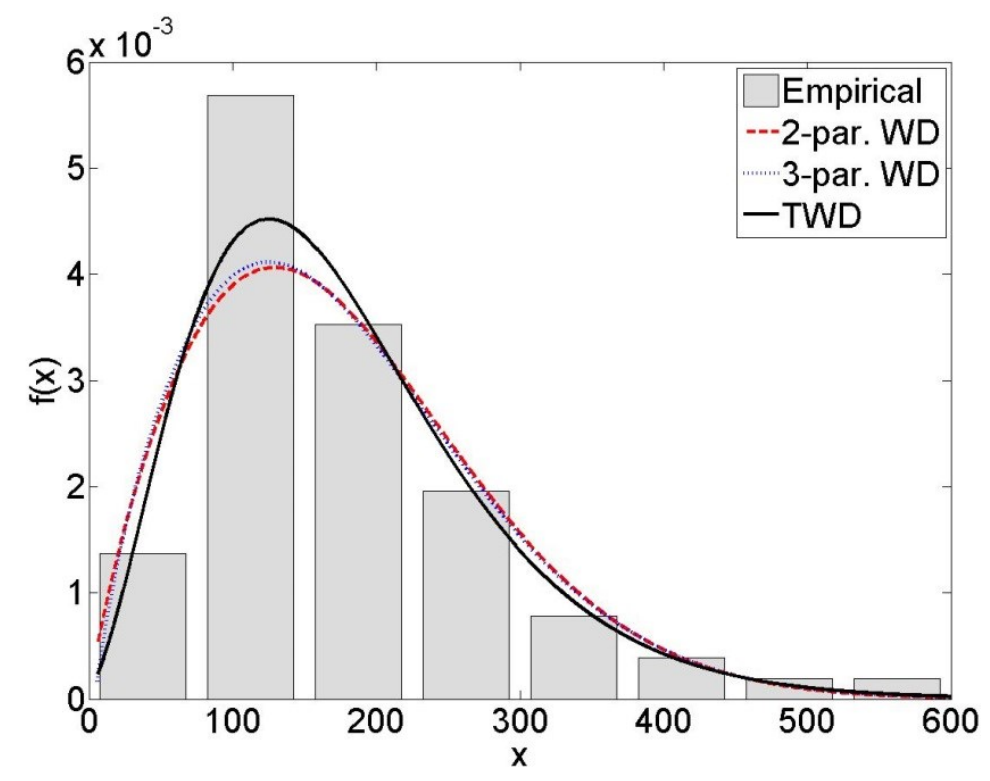

Fig. 2. Histogram of data set 2 and the fitted PDFs

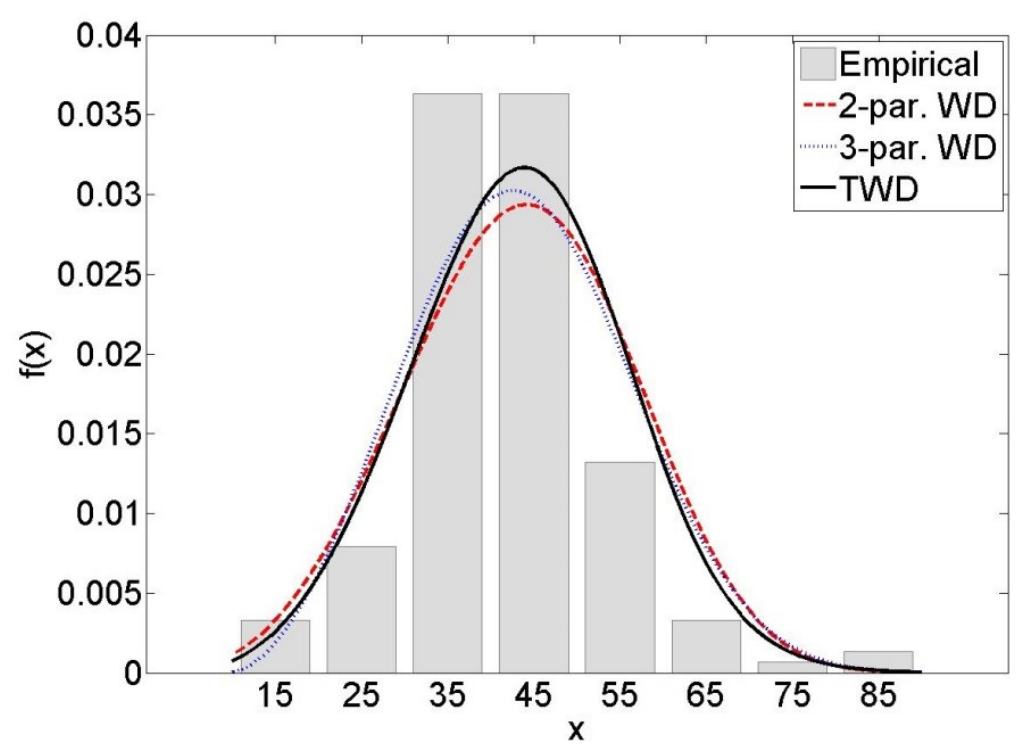

Fig. 3. Histogram of data set 3 and the fitted PDFs 


\section{Conclusion}

In this paper we studied modification of the Weibull distribution, called the transmuted Weibull distribution. The usefulness of this distribution for modelling lifetime was illustrated using three real data sets. We compared the performance of the transmuted Weibull distribution with performances of the 2-parameter and the 3-parameter Weibull distributions. Comparison of distributions was based on criteria as the Akaike's information criterion, the corrected Akaike's information criterion, the Bayesian information criterion, the coefficient of determination and the root mean square error. We demonstrated that the transmuted Weibull distribution is more flexible and it better models lifetime data of animate (breast tumour patients, infected guinea pigs) and inanimate (Kevlar/epoxy strands) objects than the 2-parameter or the 3-parameter Weibull distribution.

This paper was supported by the Slovak Grant Agency VEGA through the project No. 1/0812/17.

\section{References}

1. W. Weibull, A statistical distribution function of wide applicability. Journal of Applied Mechanics 18 (3), 293-297 (1951)

2. H. Rinne, The Weibull distribution: a handbook. (Chapman \& Hall, CRC Press, 2008)

3. H. Pham, C. D. Lai, On recent generalizations of the Weibull distributions. IEEE Transactions on Reliability 56 (3), 454-458 (2007)

4. W T. Shaw, I. R. Buckley, The alchemy of probability distributions: beyond GramCharlier expansions and a skew kurtostic-normal distribution from a rank transmutation map. Research report (2007)

5. G. R. Aryal, Ch. P. Tsokos, On the transmuted extreme value distribution with application. Nonlinear Alalysis: Theory, Methods and Applications 71, 1401-1407 (2009)

6. F. Merovci, Transmuted Rayleigh Distribution. Austrian Journal of Statistics 42 (1), 21-31 (2013)

7. M. S. Khan, R. King, Transmuted Modified Weibull Distribution: A Generalization of the Modified Weibull Probability Distribution. European Journal of Pure and Apllied Mathematics 6, 66-88 (2013)

8. M. S. Khan, R. King, R. Hudson, Characterization of the transmuted inverse Weibull Distribution. ANZIAM 55, 197-217, (2013)

9. F. Merovci, L. Puka, Transmuted Pareto Distribution. ProbStat Forum 7, 1-11 (2014)

10. G. R. Aryal, Ch. P. Tsokos, Transmuted Weibull Distribution: A Generalization of the Weibull Probability Distribution. European Journal of Pure and Apllied Mathematics 4 (2), 89-102 (2011)

11. H. Akaike, A new look at the statistical model identification, Automatic Control, IEEE Transactions 19 (6), 716-723 (1974)

12. C. M. Hurvich, C. L. Tsai, Regression and time series model selection in small samples. Biometrika 76 (2), 297-307 (1989)

13. G. Schwarz, Estimating the dimension of a model, Annals of Statistics 6 (2), 461-464, (1978)

14. R. E. Barlow, R. H. Toland, T. Freeman, A Bayesian analysis of stress rupture life of Kevlar 49/epoxy spherical pressure vessels. Procedings conference on applications of statistics, Marcel dekker, New York (1984) 
15. D. F. Andrews, A. M. Herzberg, Data: A Collection Of Problem from Many Fields for the Student and Researcher Worker. (Springer Series in Statistics, New York, 1985)

16. I. B. Abdul-Moniem, Transmuted Burr Type III Distribution. Journal of Statistics: Advances in Theory and Applications 14 (1), 37-47 (2015)

17. E. A. Owoloko, P. E. Oguntunde, A. O. Adejumo, Performance rating of the transmuted exponential distribution: an analytical approach. Springer Plus 4, 818 (2015)

18. T. Bjerkedal, Acquisition of resistance in guinea pigs infected with different doses of virulent tubercile bacilli. American Journal of Hygiene 72, 100-148 (1960)

19. E. A. Owoloko, P. E. Oguntunde, A. O. Adejumo, A Comparative Analysis on the Performance of the Convoluted Exponencial Distribution and the Exponential Distribution in terms of Flexibility. Journal of Mathematics and Statistics 12 (1) (2016)

20. M. M. Mansour, E. M. Abd Elrazik, M. S. Hamed, S. M. Mohamed, A New Additive Weibull Distribution: Based on a New Method for Adding Parameter to a Family Distributions. International Journal of Applied Mathematical Sciences 8 (1), 01-54 (2015) 